\title{
Nonsmooth control design for stabilizing switched linear systems by left eigenstructure assignment
}

\author{
Yashar Kouhi* Naim Bajcinca*,** \\ * Max Planck Institute for Dynamics of Complex Technical Systems \\ Systems and Control Theory, Sandtorstr 1, 39106 Magdeburg \\ ** Corresponding author: bajcinca@mpi-magdeburg.mpg.de
}

\begin{abstract}
Novel design algorithms for exponential stability of switched linear systems using the left eigenstructure assignment approach by state feedback control are proposed in this article. For given switching constraints in the state space $\mathbb{R}^{n}$, a state feedback controller for single-input switched systems is designed, based on the fact that closed loop system solutions are enforced to converge towards the invariant hyperplane attractor in $\mathbb{R}^{n}$, as defined by the imposed common left eigenvector. The latter is appropriately constructed to guarantee the simultaneous stabilization of all constituent linear sub-systems, and to avoid the intersection of the switching manifolds with its invariant attractor set. For arbitrary switching, a set of $n$ linearly independent common left eigenvectors in $\mathbb{R}^{n}$ is selected, introducing $n$ hyperplane attractors, and $n(n-1)$ switching control hyperplane manifolds. The attrators are sequentially "turned on", in accordance with the switching event generated by the Filippov solutions upon hitting the underlying control manifolds. Thereby, an additional state feedback control action for a second input is designed, using the nonsmooth Lyapunov stability criteria to avoid sliding modes and guarantee exponential stability for the Filippov solutions.
\end{abstract}

Keywords: Switched linear systems, nonsmooth analysis, Lyapunov function, generalized Lie derivative, left eigenstructure assignment

\section{INTRODUCTION}

The study of the stability properties of switched systems in general gives rise to a number of interesting and challenging mathematical problems [1]. A widely used approach in the stability analysis for arbitrary switching signals employs the concept of the common Lyapunov function that decreases in time along the solutions of the underlying dynamical system. It is a fact that the existence of a common smooth Lyapunov function is necessary and sufficient for the exponential stability of switched linear systems under arbitrary switching signals. Different specific classes of smooth Lyapunov functions and related converse theorems have been proposed in the literature, including the common quadratic Lyapunov function. In particular, non-quadratic or/and nonsmooth Lyapunov functions have been appealing recently, see [2]. Lyapunov based techniques have been predominant also in stabilization of switched linear systems. For instance, the local state feedback control problem for the composition of a common quadratic Lyapunov function can be transformed into a set of linear matrix inequalities, [3]. Additionally, a time dependent control switching law between the modes of a switching system is used in maintaining the Lyapunov stability condition along the system solutions, see e.g. [4].

In this work, the concept of common left eigenvector assignment is introduced as a tool for both analysis and synthesis of switched linear systems. It turns out that for all solutions of a switched linear system with sta- ble constituents sharing a commomn left eigenvector $w$, $\lim _{t \rightarrow \infty} w^{T} x(t)=0$. Hence, an important related issue consists in the computation of a single proper eigenvector in $\mathbb{R}^{n}$ which guarantees the simultaneous stability of all linear constituents at hand. To this end, tools for constructing a suitable eigenvector that stabilizes a given single linear system are pre-requisite, see $[5,6]$. This is thoroughly discussed in Section 2. Assuming that the corresponding eigenvalue is stable, the system solutions are ultimately attracted by the invariant hyperplane set $w^{T} x=0$. The idea is then to construct an invariant attractor by a proper selection of the left-eigenvector $w$, such that the latter is disjoint (up to an $\varepsilon$-Ball) with the given switching manifolds in the state-space. This is elaborated in Section 3, where formally a related sufficient condition for the stabilizability of single-input switched systems is proposed. In Section 4, this approach is generalized for the stabilization of two-input systems with arbitrary switching signals. Here, a set of $n$ independent left eigenvectors $w_{i} \in$ $\mathbb{R}^{n}, i \in\{1, \ldots, n\}$ is required, imposing additional $n(n-1)$ switching control constraints in $\mathbb{R}^{n}$. The stability of the linear sub-systems is not mandatory here. The first input is utilized to impose the underlying left eigenvector $w_{i}$ to the closed loop of sub-systems, while the second one is designed to guarantee the exponential stability of Filippov solutions using the concept of the set-valued Lie-derivative for the locally Lipschitz piecewise quadratic nonsmooth Lyapunov function defined by $V(x)=\frac{1}{2} \max \left(w_{i}^{T} x\right)^{2}$. 


\section{LEFT EIGENVECTOR ASSIGNMENT}

Consider the general state space representation of an LTI system with single input

$$
\dot{x}=A x+b u,
$$

where $x \in \mathbb{R}^{n}$ and $u \in \mathbb{R}$ are the state and the control input, respectively. Suppose we want to construct a state feedback $u=k^{T} x$ which would assign a given left eigenvector $w \in \mathbb{R}^{n}$ and a corresponding eigenvalue $\lambda_{0} \in \mathbb{R}_{<0}$ to the closed loop system. Then, by simple computation

$$
w^{T}\left(A+b k^{T}\right)=\lambda_{0} w^{T} \Longrightarrow k^{T}=-\frac{w^{T}\left(A-\lambda_{0} I\right)}{b^{T} w} .
$$

where $b^{T} w \neq 0$ is assumed, and $I$ stands for the unity matrix of order $n$. The left eigenvector $w$ appears nonlinearly in the resulting closed loop system matrix

$$
A_{\mathrm{cl}}=\left(I-\frac{b w^{T}}{b^{T} w}\right) A+\lambda_{0} \frac{b w^{T}}{b^{T} w} .
$$

A next natural problem arising from the closed loop description in (3) consists in exploring the proper selection of $w$ and $\lambda_{0}$ such that $A_{\mathrm{cl}}=A+b k^{T}$ becomes a Hurwitz matrix. Therefore, let $p(\lambda)=\operatorname{det}(\lambda I-A)$ be the characteristic polynomial of the open loop system

$$
p(\lambda)=\lambda^{n}+a_{1} \lambda^{n-1}+\ldots+a_{n},
$$

and $q(\lambda)=\operatorname{det}\left(\lambda I-A_{\mathrm{cl}}\right)$ the characteristic polynomial of the closed loop matrix (3)

$$
q(\lambda)=\lambda^{n}+\alpha_{1} \lambda^{n-1}+\ldots+\alpha_{n} .
$$

The relationship between the coefficients $\alpha_{i}$ and $a_{i}, i=$ $1, \ldots, n$ is given by the Bass-Gura formula, see [7]

$$
\begin{aligned}
& \alpha_{1}=a_{1}-k^{T} b, \\
& \alpha_{2}=a_{2}-k^{T} A b-a_{1} k^{T} b, \\
& \alpha_{3}=a_{3}-k^{T} A^{2} b-a_{1} k^{T} A b-a_{2} k^{T} b,
\end{aligned}
$$

and so forth. On the other hand,

$$
\begin{aligned}
\operatorname{det}(\lambda I & \left.-A-b k^{T}\right)= \\
& =\operatorname{det}(\lambda I-A) \operatorname{det}\left[I-(\lambda I-A)^{-1} b k^{T}\right] \\
& =p(\lambda)\left[1-k^{T}(\lambda I-A)^{-1} b\right] \\
& =p(\lambda)\left[1+\frac{w^{T}\left(A-\lambda_{0} I\right)(\lambda I-A)^{-1} b}{b^{T} w}\right] \\
& =p(\lambda)\left[\frac{w^{T}\left(\lambda I-A-\lambda_{0} I+A\right)(\lambda I-A)^{-1} b}{b^{T} w}\right] \\
& =\left(\lambda-\lambda_{0}\right) \frac{b^{T} \operatorname{adj}\left(\lambda I-A^{T}\right) w}{b^{T} w} .
\end{aligned}
$$

[Hint: For the above derivation steps the reader is referred to Section 3.2 in [7].] The latter expression reveals the fact that $\lambda_{0}$ is an eigenvalue of $A_{\mathrm{cl}}$, while the rest $n-1$ eigenvalues, being the roots of the polynomial

$$
\frac{b^{T} \operatorname{adj}\left(\lambda I-A^{T}\right) w}{b^{T} w}=\lambda^{n-1}+\beta_{1} \lambda^{n-2} \ldots+\beta_{n-1},
$$

are uniquely specified by the left eigenvector $w$. The coefficients $\beta_{i}, i=1, \ldots, n-1$ are computed by comparing the coefficients of the following polynomial identity

$$
\begin{aligned}
\lambda^{n}+\alpha_{1} \lambda^{n-1} & \ldots+\alpha_{n}= \\
& =\left(\lambda-\lambda_{0}\right)\left(\lambda^{n-1}+\beta_{1} \lambda^{n-2} \ldots+\beta_{n-1}\right),
\end{aligned}
$$

yielding

$$
\beta_{i}=\frac{b^{T}\left(a_{i} I+a_{i-1} A+\ldots+A^{i}\right)^{T} w}{b^{T} w} .
$$

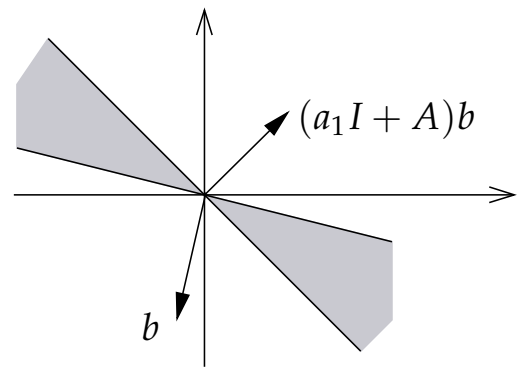

Fig. 1. Selection of the left eigenvector for $n=2$. The gray shaded area depicts the region of all stabilizing common left eigenvectors $w$.

Example 1. Due to the nonlinear appearance of the eigenvector $w$ in (8), applying the Routh-Hurwitz stability criteria for the construction of $w$ is, in general, tedious. However, for $n=2$ and $n=3$, we can obtain simple solutions with an insightful geometrical illustration. For instance, for $n=2$, from (8) it follows

$$
\beta_{1}=\frac{b^{T}\left(a_{1} I+A\right)^{T} w}{b^{T} w}>0 .
$$

This inequality is held if the inner-products of $w$ with $b$ and $\left(a_{1} I+A\right) b$ share the same sign. Hence, the set of all eigenvectors is defined by the shaded area in Figure 1. If $n=3$, we additionally need

$$
\beta_{2}=\frac{b^{T}\left(a_{2} I+a_{1} A+A^{2}\right)^{T} w}{b^{T} w}>0 .
$$

In the following we provide a systematic method for the design of all stabilizing left eigenvectors $w$ in (3). For the sake of simplicity, consider first, the canonical controller form representation of the system (1)

$$
\dot{x}=A_{c} x+b_{c} u
$$

with

$$
A_{c}=\left(\begin{array}{ccccc}
0 & 1 & 0 & \ldots & 0 \\
0 & 0 & 1 & \ldots & 0 \\
\cdot & & & & \\
\cdot & & & & \\
\cdot & 0 & 0 & \ldots & 1 \\
0 & 0 & -a_{n-2} & \ldots & -a_{1}
\end{array}\right), b_{c}=\left(\begin{array}{c}
0 \\
0 \\
\cdot \\
\cdot \\
\cdot \\
0 \\
1
\end{array}\right) .
$$

Let $w_{c}=\left[w_{c, 1}, \ldots, w_{c, n-1}, 1\right]^{T}$ and $\lambda_{0}$ be the desired left eigenvector and its eigenvalue, respectively. Observe that w.l.o.g. we can set $w_{c, n}=1$, to assure the mandatory condition $1=b_{c}^{T} w_{c} \neq 0$. It is an easy exercise to show now that the state feedback $u=k_{c}^{T} x$ [in accordance with (2)], with $k_{c}^{T}=-w_{c}^{T}\left(A_{c}-\lambda_{0} I\right)$, leads to the closed loop system matrix $A_{c, \mathrm{cl}}=A_{c}+b k_{c}^{T}$ given by

$$
A_{c, \mathrm{cl}}=\left(I-\frac{b_{c} w_{c}^{T}}{b_{c}^{T} w_{c}}\right) A_{c}+\lambda_{0} \frac{b_{c} w_{c}^{T}}{b_{c}^{T} w_{c}} .
$$

[For convenience, with regard to the comparison with (14), we keep here the term $b_{c}^{T} w_{c}=1$ in the denominator.], that does not depend on the parameters $a_{1}, \ldots, a_{n}$ of the matrix $A_{c}$, as revealed by its explicit form

$$
A_{c, \mathrm{cl}}=\left(\begin{array}{cccc}
0 & 1 & \ldots & 0 \\
0 & 0 & \cdots & 0 \\
\vdots & & & \\
0 & 0 & & 1 \\
\lambda_{0} w_{c, 1} & -w_{c, 1}+\lambda_{0} w_{c, 2} & \ldots & -w_{c, n-1}+\lambda_{0}
\end{array}\right) .
$$


Then, it follows

$$
\begin{aligned}
& \operatorname{det}\left(\lambda I-A_{c, \mathrm{cl}}\right)= \\
& \quad=\lambda^{n}+\left(w_{c, n-1}-\lambda_{0}\right) \lambda^{n-1}+\ldots-\lambda_{0} w_{c, 1} \\
& \quad=\left(\lambda-\lambda_{0}\right)\left(\lambda^{n-1}+w_{c, n-1} \lambda^{n-2}+\ldots+w_{c, 2} \lambda+w_{c, 1}\right) .
\end{aligned}
$$

Hence, with $\Lambda=\left[1, \lambda, \ldots, \lambda^{n-1}\right]^{T}$, the characteristic polynomial of the closed loop can be written as

$$
q_{c}(\lambda)=\left(\lambda-\lambda_{0}\right) \Lambda^{T} w_{c} .
$$

Each controllable system (1) can be converted into the controller canonical form using the transformation $T=$ $\Phi_{c} \Phi_{c c}^{-1}$, where $\Phi_{c}$ and $\Phi_{c c}$ are the controllability matrices of the original and of the transformed system, respectively. Then, $A_{c}=T^{-1} A T$ and $b_{c}=T^{-1} b$ applies, and we get

$$
\begin{aligned}
T^{-1} A_{\mathrm{cl}} T & =T^{-1} A T-\frac{\left(T^{-1} b\right) w^{T} A T}{b^{T} w}+\lambda_{0} \frac{\left(T^{-1} b\right) w^{T} T}{b^{T} w} \\
& =\left(I-\frac{b_{c}\left(w^{T} T\right)}{b_{c}^{T}\left(T^{T} w\right)}\right) A_{c}+\lambda_{0} \frac{b_{c}\left(w^{T} T\right)}{b_{c}^{T}\left(T^{T} w\right)} .
\end{aligned}
$$

A comparison of this expression with (12), implies that the condition $T^{-1} A_{\mathrm{cl}} T=A_{c, \mathrm{cl}}$ holds if $w^{T}=\gamma \cdot w_{c}^{T} T^{-1}$, where $\gamma$ is any nonzero real scalar. This closes the proof of the following statement.

Theorem 1. Let the single-input LTI system (1) be controllable, and define $T=\Phi_{c} \Phi_{c c}^{-1}$. Assume $\lambda_{0}<0$, and consider any Hurwitz polynomial in the form (13). Then, the closed loop matrix (3) is Hurwitz, if the left eigenvector $w$ is given by $w=\gamma \cdot\left(T^{-1}\right)^{T} w_{c}$, for some real nonzero $\gamma$, and $w_{c}=\left[\begin{array}{lll}w_{c, 1} \ldots w_{c, n-1} & 1\end{array}\right]^{T}$.

\section{STATE-SPACE SWITCHING CONSTRAINTS}

\subsection{Stability analysis}

Let $\mathcal{A}=\left\{A_{1}, \ldots, A_{m}\right\}$ be a collection of Hurwitz matrices in $\mathbb{R}^{n \times n}$. In this section, we consider a switched linear system defined as

$$
\dot{x}(t)=A_{\delta(x)} x(t), \text { where } \delta: \mathbb{R}^{n} \rightarrow\{1, \ldots, m\}
$$

is a piecewise constant function, defining the active modes $\dot{x}(t)=A_{\alpha} x(t), A_{\alpha} \in \mathcal{A}$. The function $\delta$ can be associated a switching constraint set

$$
\mathcal{M}=\left\{x \in \mathbb{R}^{n} ; M(x)=0\right\}
$$

where $M(x)=0$ represents a switching manifold between two different modes in $\mathcal{A}$. For a given switching function $\delta(x)$, and a fixed initial condition $x(0)$, the solution of the switched system (15) is uniquely given by

$$
x(t)=e^{A_{\delta_{k}}\left(t-t_{k}\right)} \ldots e^{A_{\delta_{1}}\left(t_{2}-t_{1}\right)} x(0),
$$

where $t>t_{k}>\ldots>t_{1}>0$ refer to the switching times, and $\delta_{i}=\delta\left(x\left(t_{i}\right)\right), i=1, \ldots, k$.

Consider now the special case with all matrices in $A_{\alpha} \in \mathcal{A}$, sharing a same left eigenvector $w$. Left-side multiplication of equation (17) by $w^{T}$ implies

$$
w^{T} x(t)=e^{\lambda_{0, k}\left(t-t_{k}\right)} \ldots e^{\lambda_{0,1}\left(t_{2}-t_{1}\right)} w^{T} x(0),
$$

where we use the fact that $w^{T}$ is a left eigenvector of the matrix $e^{A_{\delta_{i}} t}$, too. Hence, if all eigenvalues $\lambda_{0, i}, \quad i=$ $1, \ldots, k$ are stable, then

$$
\lim _{t \rightarrow \infty} w^{T} x(t)=0
$$

and, consequently, all solutions of the switched system (15) [independently of the initial value $x(0)$ ] converge to the invariant set

$$
\mathcal{X}=\left\{x \in \mathbb{R}^{n} \mid w^{T} x=0\right\},
$$

as revealed by

$$
x \in \mathcal{X} \Rightarrow w^{T} \dot{x}=\lambda_{0, i} w^{T} x=0 .
$$

[Note that all solutions, starting from $\mathcal{X}$ at $t=0$, remain in $\mathcal{X}$ for all $t>0$.] Moreover, according to equation (18) for all $t$ and for all $i=1, \ldots, k$

$$
\left\|w^{T} x\right\| \leq e^{\max \left(\lambda_{0, i}\right) t}\left\|w^{T} x(0)\right\|,
$$

implying that the solutions (17) converge to $\mathcal{X}$ exponentially fast. In other words, $\mathcal{X}$ is an invariant attractor set. Lemma 1. Consider the switched system (15), and suppose that $\mathcal{X}$ and $\mathcal{M}$, as defined by (19) and (16), respectively, are disjoint in the sense that $(\mathcal{X}+\varepsilon \mathbb{B}) \cap \mathcal{M}=\emptyset$ for some $\varepsilon>0$, where $\mathbb{B}$ represents a closed unit ball in $\mathbb{R}^{n}$. Then, if all matrices in $\mathcal{A}$ share a common left eigenvector $w$, the system (15) is exponentially stable.

Proof: Referring to the conditions of the theorem, all solutions of the underlying switched system enter the set $(X+\varepsilon \mathbb{B})$ in a finite time. Since no switching takes place thereafter, the system (15) reduces to a linear system of the form $\dot{x}=A_{\alpha} x$ for some $A_{\alpha} \in \mathcal{A}$, which is, by definition, exponentially stable. This completes the proof.

This lemma is introduced primarily for design purposes in the next section, but it can be utilized for stability test, as well, as illustrated by the next example.

Example 2. It is a fact that no common quadratic Lyapunov function exists for (15) if there exist matrices $R_{\alpha}=$ $R_{\alpha}^{T}$ satisfying $\sum_{\alpha=1}^{m}\left(A_{\alpha}^{T} R_{\alpha}+R_{\alpha} A_{\alpha}\right)>0$, see [1]. Consider the example taken from [3], with slightly modified matrices $A_{\alpha}$ and $R_{\alpha}, \alpha \in\{1,2\}$

$$
\begin{gathered}
A_{1}=\left(\begin{array}{ccc}
-1 & 0 & 0 \\
0 & -1 & -1 \\
0 & 1 & -1
\end{array}\right) A_{2}=\left(\begin{array}{ccc}
-3 & 0 & 0 \\
0 & -1 & -10 \\
0 & 0.1 & -1
\end{array}\right) \\
R_{1}=\left(\begin{array}{ccc}
-1 & 0 & 0 \\
0 & 0.299 & 0.704 \\
0 & 0.704 & 2.470
\end{array}\right) R_{2}=\left(\begin{array}{ccc}
-1 & 0 & 0 \\
0 & 0.212 & -0.553 \\
0 & -0.553 & 1.971
\end{array}\right) .
\end{gathered}
$$

Note that these matrices satisfy the latter inequality, hence, no statement about the stability of the system based on the concept of common quadratic Lyapunov function can be made here. However, with $\mathcal{M}=\{x \in$ $\left.\mathbb{R} ; x_{2}^{2}+x_{3}^{2}-a x_{1}^{2}+1=0, a \in \mathbb{R}_{>0}\right\}$, the underlying switched system is exponentially stable since $(i) w=\left[\begin{array}{ll}1,0,0 & 0\end{array}\right]^{T}$ is a left common eigenvector for both individuals $A_{1}$ and $A_{2}$, (ii) $A_{1}$ and $A_{2}$ are Hurwitz, and (iii) $(\mathcal{X}+\varepsilon \mathbb{B}) \cap$ $\mathcal{M}=\emptyset$ for $0 \leq \epsilon<\min (1,1 / \sqrt{a})$ and the invariant set $\mathcal{X}=\left\{x \in \mathbb{R} ; x_{1}=0\right\}$.

\subsection{Control design}

Consider a single input switched system

$$
\dot{x}(t)=A_{\delta} x(t)+b_{\delta} u(t),
$$

with $\delta=\delta(x)$ defined as in the previous section, [for notation simplicity, we drop here its explicit dependency on $x]$, and

$$
A_{\delta} \in \mathcal{A}=\left\{A_{1}, \ldots, A_{m}\right\}, b_{\delta} \in \mathcal{B}=\left\{b_{1}, \ldots, b_{m}\right\} .
$$

Consider exponential stabilization of $(21)$ by means of a local state feedback controller $u=k_{\delta}^{T} x$. In our control strategy, $k_{\delta}$ is requried to impose the same common left eigenvector $w$ and a corresponding eigenvalue $\lambda_{0}$ to all 


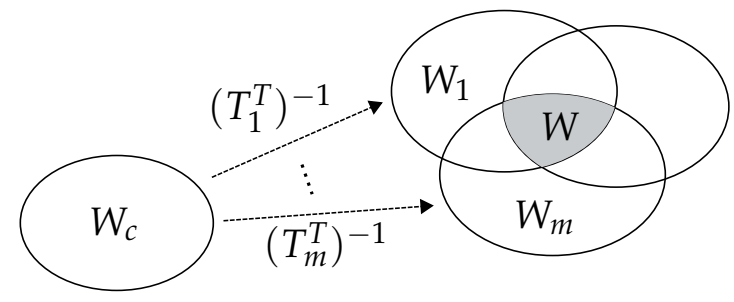

Fig. 2. Selection of a proper common left eigenvector in accordance with the conditions (i)-(ii) of Theorem 2 .

closed loop matrices $A_{\mathrm{cl}, \alpha}=A_{\alpha}+b_{\alpha} k_{\alpha}^{T}, \alpha \in\{1, \ldots, m\}$. To this end, use (2) and a $w$ such that $b_{\alpha}^{T} w \neq 0, b_{\alpha} \in \mathcal{B}$

$$
k_{\delta}^{T}=-\frac{w^{T}\left(A_{\delta}-\lambda_{0} I\right)}{b_{\delta}^{T} w} .
$$

Note that $k_{\delta}$ is itself a switching control gain, taking the values in $\mathcal{K}=\left\{k_{1}, \ldots, k_{m}\right\}$, where $k_{\alpha}=k_{\delta}$ for $\delta(x)=\alpha \in\{1, \ldots, m\}$. The critical point here consists, however, in the appropriate design of $w$, such that (21) is stabilized by $u=k_{\delta}^{T} x$. The following theorem, which is a direct consequence of Lemma 1, provides a corresponding design roadmap.

Theorem 2. If $w \in \mathbb{R}$ and $\lambda_{0}<0$ exist, such that

(i) $w^{T} A_{1}=\ldots=w^{T} A_{m}=\lambda_{0} w^{T}$,

(ii) $A_{\mathrm{cl}, \alpha}=A_{\alpha}+b_{\alpha} k_{\alpha}^{T}$ are Hurwitz for $\alpha \in\{1, \ldots, m\}$, with $k_{\alpha} \in \mathcal{K}$, and

(iii) $(\mathcal{X}+\varepsilon \mathbb{B}) \cap \mathcal{M}=\emptyset$ for some $\varepsilon>0$, where $\mathcal{X}=\{x \in$ $\left.\mathbb{R}^{n} ; w^{T} x=0\right\}$

then the switched system (21) is globally exponentially stabilizable by the feedback law $u=k_{\delta}^{T} x$, with $k_{\delta}$ given by $(23)$.

A basic approach for constructing a proper common left eigenvector $w$ up to a scaling factor $\gamma \neq 0$, such that the conditions $(i)$-(ii) of this theorem are fulfilled, is schematically depicted in Figure 2. Let $W_{c}$ be the space of all $w_{c} \in \mathbb{R}^{n}$ that fulfill (13), for all eigenvalues $\lambda_{1}, \ldots, \lambda_{n-1}$ that lie in the open left half of the complex plane, and define $W_{\alpha}=\left(T_{\alpha}^{T}\right)^{-1} W_{c}=\left\{\left(T_{\alpha}^{T}\right)^{-1} w_{c} ; w_{c} \in W_{c}\right\}, \alpha \in$ $\{1, \ldots, m\}$. Then, any vector $w$ from $W=W_{1} \cap \ldots \cap$ $W_{m}$, such that $b_{\alpha}^{T} w \neq 0$ holds for every $\alpha$, fulfills (i)(ii) in Theorem 2. Furthermore, in order to guarantee the exponential stability of the closed loop of (21) [referring to the condition (iii) of the theorem], one has to pick up such a vector $w \in W$ that guarantees the disjunction condition $(\mathcal{X}+\varepsilon \mathbb{B}) \cap \mathcal{M}=\emptyset$.

\section{ARBITRARY SWITCHING CONSTRAINTS}

We start the section by recalling some definitions and results which are used in the sequel. For more details, the reader is referred to $[8,9]$.

\subsection{Preliminaries}

A differential inclusion is defined by

$$
\dot{x} \in F(x),
$$

where $F: \mathcal{G} \rightarrow \mathcal{P}\left(\mathbb{R}^{n}\right)$ is a set-valued map, $\mathcal{G}$ is an open subset of $\mathbb{R}^{n}$, and $\mathcal{P}\left(\mathbb{R}^{n}\right)$ represents the power set of $\mathbb{R}^{n}$. Differential inclusions are instrumental for the analysis of discontinuous and uncertain systems. Diverse concepts for the solutions of differential inclusions exist. In particular, we consider here the Filippov solutions, which arise by approximating a discontinuous vector field $X: \mathcal{G} \rightarrow \mathbb{R}^{n}$ in $\dot{x}=X(x)$ by the Filippov set-valued map defined by

$$
F(x):=\cap_{\delta>0} \cap_{\mu(S)=0} \overline{\operatorname{co}}\{X(\mathcal{B}(x, \delta) \backslash S)\},
$$

where $\overline{\mathrm{co}}$ denotes convex closure, $\mu$ denotes the Lebesgue measure, $S$ is the set of measured zero points, and $\mathcal{B}(x, \delta) \subset \mathbb{R}^{n}$ is a ball with center at $x \in \mathbb{R}^{n}$ and radius $\delta$.

The generalized gradient of a locally Lipschitz function $V: \mathbb{R}^{n} \rightarrow \mathbb{R}$ at the point $x \in \Omega$ denoted by $\partial V(x)$ is defined as

$$
\partial V(x):=\operatorname{co}\left\{\lim _{i \rightarrow \infty} \nabla V\left(x_{i}\right) ; x_{i} \rightarrow x, x_{i} \notin \Omega \cup S\right\},
$$

where "co" stands for the convex hull, and $\Omega$ represents a manifold in $\mathbb{R}^{n}$, where the gradient $\nabla V(x)$ is discontinouos. The set-valued Lie derivative of the latter function $V(x)$ with respect to a set valued map $F$ denoted by $\tilde{L}_{F} V(x)$, is defined as

$$
\tilde{L}_{F} V(x)=\left\{a \in \mathbb{R} ; \exists \nu \in F(x), \zeta^{T} \nu=a, \forall \zeta \in \partial V(x)\right\} .
$$

If $F$ is convex and compact, then, for all $x \in \mathbb{R}^{n}, \tilde{L}_{F} V(x)$ is a closed and bounded interval in $\mathbb{R}$, possibly empty. If $V$ is continously differentiable at $x$ then

$$
\tilde{L}_{F} V(x)=\left\{(\nabla V(x))^{T} \nu ; \nu \in F(x)\right\} .
$$

Recall that the usefulness of the concept of the Lie derivatives stems from the fact that it enables us to study the evolution of the function $V$ along the solutions of a differential inclusion $\dot{x} \in F(x)$, without the explicit need for the solution of the latter. The following theorem implies the stability of Filippov solutions to the differential inclusion (24) for the nonsmooth locally Lipschitz Lyapunov function $V=V(x)$, see $[8,9]$.

Theorem 3. Consider the Filippov set valued map $F: \mathcal{G} \rightarrow$ $\mathcal{P}\left(\mathbb{R}^{n}\right)$ and a corresponding differential inclusion $\dot{x} \in F(x)$. Let $x=0$ be the only equilibrium of the differential inclusion. Then, $x=0$ is a globally asymptotically stable equilibrium of $\dot{x} \in F(x)$, if a function $V: \mathbb{R}^{n} \rightarrow \mathbb{R}$ exists, such that

(i) $V$ is locally Lipschitz and regular on $\mathbb{R}^{n}$,

(ii) $V(0)=0$ and $V(x)>0$ for each $x \in \mathbb{R}^{n} \backslash\{0\}$, and

(iii) $\max \tilde{L}_{F} V(x)<0$ for each $x \in \mathbb{R}^{n}$.

\subsection{Control design}

Consider a switching linear system with two inputs

$$
\dot{x}=A_{\sigma} x(t)+b_{1, \sigma} u_{1}(t)+b_{2, \sigma} u_{2}(t),
$$

where $\sigma: \mathbb{R}_{\geq 0} \rightarrow\{1, \ldots, m\}$ is an arbitrary timedependent piecewise continuous switching function, and

$$
A_{\sigma} \in \mathcal{A}=\left\{A_{1}, \ldots, A_{m}\right\}, \quad b_{k, \sigma} \in \mathcal{B}_{k}=\left\{b_{k, 1}, \ldots, b_{k, m}\right\},
$$

for $k=1,2$. Note that the switching function $\sigma$ infers a larger class of switched systems in comparison with the state-dependent function $\delta$ in the previous section.

Consider the following control Lyapunov function

$$
V(x)=\frac{1}{2} \max \left(w_{i}^{T} x\right)^{2}, i \in\{1, \ldots, n\},
$$

where $w_{i}, i \in\{1, \ldots, n\}$, are any linearly independent vectors in $\mathbb{R}^{n}$, and $b_{1, \alpha}^{T} w_{i} \neq 0$, for all $\alpha \in\{1, \ldots, m\}$. It is obvious that this function is locally Lipschitz and regular. Also, it is positive for $x \neq 0$, and $V(x)=0 \Rightarrow x=0$. Therefore, the function $V(x)$ fulfills the conditions $(i)$ and 
(ii) of Theorem 3. Moreover, it is differentiable almost everywhere, but on the hyperplane manifolds

$$
\mathcal{X}_{i j}=\left\{x \in \mathbb{R}^{n} ;\left(w_{i}-w_{j}\right)^{T} x=0 \vee\left(w_{i}+w_{j}\right)^{T} x=0\right\},
$$

where $\left(w_{i}^{T} x\right)^{2}=\left(w_{j}^{T} x\right)^{2}=\max \left\{\left(w_{k}^{T} x\right)^{2}, k=1, \ldots, n\right\}$. The objective of our control problem consists in designing the appropriate controllers for the inputs $u_{1}$ and $u_{2}$, such that the control Lyapunov function in (30) fulfills the last condition (iii) in Theorem 3 along the Filippov solutions of the underlying closed loop system.

To this end, consider the set given by

$$
\Omega_{i}=\left\{x \in \mathbb{R}^{n} ;\left(w_{i}^{T} x\right)^{2} \geq\left(w_{j}^{T} x\right)^{2}, j=1, \ldots, n\right\},
$$

and define the control $u_{1}=k_{1, \sigma i}^{T} x$ for $x \in \Omega_{i}$, such that the closed loop

$$
A_{\mathrm{cl}, \sigma i}=A_{\sigma}+b_{1, \sigma} k_{1, \sigma i}^{T}+b_{2, \sigma} k_{2, \sigma i}^{T},
$$

is assigned the left eigenvector $w_{i}$. According to (2)

$$
k_{1, \sigma i}^{T}=-\frac{w_{i}^{T}\left(A_{\sigma}+b_{2, \sigma} k_{2, \sigma i}^{T}-\lambda_{0} I\right)}{b_{1, \sigma}^{T} w_{i}} .
$$

The resulting closed loop system matrix then reads

$$
A_{\mathrm{cl}, \sigma i}=\tilde{A}_{\sigma i}+\tilde{b}_{\sigma i} k_{2, \sigma i}^{T}
$$

where

$$
\begin{gathered}
\tilde{A}_{\sigma i}=\left(I-\frac{b_{1, \sigma} w_{i}^{T}}{b_{1, \sigma}^{T} w_{i}}\right) A_{\sigma}+\lambda_{0} \frac{b_{1, \sigma} w_{i}^{T}}{b_{1, \sigma}^{T} w_{i}}, \\
\tilde{b}_{\sigma i}=\left(I-\frac{b_{1, \sigma} w_{i}^{T}}{b_{1, \sigma}^{T} w_{i}}\right) b_{2, \sigma} .
\end{gathered}
$$

In our setting, the closed loop (33) experiences a switching in the following situations:

(s1) The independent switching signal $\sigma=\sigma(t)$ causes an internal switching in the open loop system matrix $A_{\sigma} \in \mathcal{A}$ in (29). Then, $k_{1, \sigma i}$ is updated using (32). This will be referred to as autonomous switching.

(s2) A solution of $\dot{x}=A_{\mathrm{cl}, \sigma i} x(t)$ for some fixed $x(0)$, falls down onto a hyperplane $\mathcal{X}_{j i}=\Omega_{j} \cap \Omega_{i}$ at some point $\xi$. Then, $k_{2, \sigma i}$ has to be updated according to the rules to be shortly conceived. Moreover, if the solution enters the set $\Omega_{i}$ from $\Omega_{j}, j \neq i, k_{1, \sigma j}$ switches to $k_{1, \sigma i}$, in accordance with (32). Notice that $\mathcal{X}_{j i}$ is a switching manifold imposed by the control.

With reference to these switching conditions, we introduce now the Filippov set valued map for the differential inclusion $\dot{x} \in F(x)$ that governs the Filippov solutions of the closed loop system $\dot{x}=A_{\mathrm{cl}, \sigma i} x(t)$ as

$F(x)=\left\{\sum_{\alpha=1}^{m} \sum_{i=1}^{n} \gamma_{\alpha i} A_{\mathrm{cl}, \alpha i} x ; \gamma_{\alpha i} \geq 0, \sum_{\alpha=1}^{m} \sum_{i=1}^{n} \gamma_{\alpha i}=1\right\}$

where $A_{\mathrm{cl}, \alpha i}=A_{\mathrm{cl}, \sigma i}$ if $\sigma(t)=\alpha \in\{1, \ldots, m\}$. If for some $t>0$, no autonomous switching happens (that is, $\sigma$ is continuous at the time instant $t$ ), then for $x \in \Omega_{i}$, it follows

$$
F(x)=A_{\mathrm{cl}, \alpha i} x \text {. }
$$

[Since $F(x)$ is a singleton, we drop the braces.] If $A_{\mathrm{cl}, \alpha i}$ switches to $A_{\mathrm{cl}, \beta j}$ due to the condition in (s1) or (s2), where $\alpha, \beta \in\{1, \ldots, m\}$ and $i, j \in\{1, \ldots, n\}$, then

$$
\begin{aligned}
& F(x)=\left\{\left(\gamma_{\alpha i} A_{\mathrm{cl}, \alpha i}+\gamma_{\beta j} A_{\mathrm{cl}, \beta j}\right) x ;\right. \\
& \left.\gamma_{\alpha i}+\gamma_{\beta j}=1, \gamma_{\alpha i}, \gamma_{\beta j} \geq 0\right\},
\end{aligned}
$$

whereby all other $\gamma$-parameters in (34) are naught. Note that, while the Filippov set-valued map, referring to an autonomous switching event, involves always two constituent modes, in the general case, a switching control manifold may call for additional ones. For instance, in the switching control manifold $\mathcal{X}_{j i k}=\Omega_{j} \cap \Omega_{i} \cap \Omega_{k}$, three active modes are involved.

Having introduced the Filippov set valued map, in the following, we elaborate different scenarios separately, and investigate for each, the condition (iii) in Theorem 3 for the resulting set-valued Lie derivative $\tilde{L}_{F} V(x)$.

Scenario (a): If no switching occurs at some $x \in \Omega_{i}$, according to the definition (30) the function $V(x)$ is continously differentiable at $x$. According to (28), the set valued Lie derivative is singleton, and the underlying condition (iii) is satisfied since

$$
\max \tilde{L}_{F} V(x)=\lambda_{0} \max \left(w_{i}^{T} x\right)^{2}<0 .
$$

Scenario (b): Consider the autonomous switching condition, as defined in (s1). Then, the closed loop matrix $A_{\mathrm{cl}, \alpha i}$ switches to $A_{\mathrm{cl}, \beta i}$, and the Filippov set valued map is given by $(36)$, with $j=i$. The function $V(x)$ is again continously differentiable at $x \in \Omega_{i}$, and referring to (28), the set valued Lie derivative reads

$$
\begin{aligned}
\tilde{L}_{F} V(x) & =\left\{\left(w_{i}^{T} x\right) w_{i}^{T}\left(\gamma_{\alpha i} A_{\mathrm{cl}, \alpha i}+\gamma_{\beta i} A_{\mathrm{cl}, \beta i}\right) x\right\} \\
& =\lambda_{0}\left(w_{i}^{T} x\right)^{2},
\end{aligned}
$$

where we make use of $w_{i}^{T} A_{\mathrm{cl}, \alpha i}=w_{i}^{T} A_{\mathrm{cl}, \beta i}=\lambda_{0} w_{i}^{T}$. Hence, (iii) holds.

Scenario (c): Consider the switching scenario, as defined in (s2), at some $\xi \in \mathcal{X}_{j i}$. [For the sake of simplicity, we confine the discussion to switching control manifolds $\mathcal{X}_{j i}$ of dimension $n-1$.] Then, $A_{\mathrm{cl}, \alpha j}$ switches to $A_{\mathrm{cl}, \alpha i}$, while the function $V(x)$ is now nonsmooth at $x=\xi$. The generalized gradient of $V$ at $\xi$ is given by

$$
\begin{aligned}
\partial V(\xi)=\left\{\left(w_{i}^{T} \xi\right)\left(\mu_{\alpha j} w_{j}^{T} \pm \mu_{\alpha i} w_{i}^{T}\right) ;\right. \\
\left.\mu_{\alpha j}+\mu_{\alpha i}=1, \mu_{\alpha j}, \mu_{\alpha i} \geq 0\right\},
\end{aligned}
$$

whereas, the Filippov set valued map reads

$$
\begin{aligned}
& F(\xi)=\left\{\left(\gamma_{\alpha j} A_{\mathrm{cl}, \alpha j}+\gamma_{\alpha i} A_{\mathrm{cl}, \alpha i}\right) \xi ;\right. \\
& \left.\gamma_{\alpha j}+\gamma_{\alpha i}=1, \gamma_{\alpha j}, \gamma_{\alpha i} \geq 0\right\} .
\end{aligned}
$$

The idea now consists in avoiding the sliding modes, as they may cause diverging Filippov solutions. Therefore, we will design a state feedback $u_{2}=k_{2, \sigma i}^{T} \xi$, such that the resulting set valued Lie derivative at $\xi \in \mathcal{X}_{j i}$ is empty, or, equivalently, such that $\max \left(\tilde{L}_{F} V(\xi)\right)=-\infty$. As a consequence, the Filippov solutions leave the switching manifold $\mathcal{X}_{j i}$ immediately upon hitting it. According to (27), a set valued Lie derivative is empty if with $\nu=\left(\gamma_{\alpha j} A_{\mathrm{cl}, \alpha j}+\right.$ $\left.\gamma_{\alpha i} A_{\mathrm{cl}, \alpha i}\right) \xi \in F(\xi)$ no $\gamma_{\alpha j}$ and $\gamma_{\alpha i}$ in (40) exist, such that $\zeta^{T} \nu=$ const holds, for any vector $\zeta=\left(w_{i}^{T} \xi\right)\left(\mu_{\alpha j} w_{j}^{T} \mp\right.$ $\left.\mu_{\alpha i} w_{i}^{T}\right) \in \partial V(\xi)$ in (39). A related necessary and sufficient condition which guarantees this situation is found to be

$$
\left(w_{i}^{T} \xi\right)\left(w_{j}^{T} \mp w_{i}^{T}\right)\left(\gamma_{\alpha j} A_{\mathrm{cl}, \alpha j}+\gamma_{\sigma i} A_{\mathrm{cl}, \alpha i}\right) \xi \neq 0 .
$$

The latter inequality is satisfied if and only if

$$
\xi^{T} A_{\mathrm{cl}, \alpha j}^{T}\left(w_{j} \mp w_{i}\right) \times\left(w_{j}^{T} \mp w_{i}^{T}\right) A_{\mathrm{cl}, \alpha i} \xi>0 .
$$

For the sake of simplicity, consider only the negative signs in the above expression. Substituting $A_{\mathrm{cl}, \alpha i}$ from (33) [with $\sigma=\alpha$ ] into the latter equation, and introducing $f(x)=x^{T} A_{\mathrm{cl}, \alpha j}^{T}\left(w_{j}-w_{i}\right)\left(w_{j}-w_{i}\right)^{T} \tilde{A}_{\alpha i} x$ and $g(x)^{T}=$ $x^{T} A_{\mathrm{cl}, \alpha j}^{T}\left(w_{j}-w_{i}\right)\left(w_{j}-w_{i}\right)^{T} \tilde{b}_{\alpha i} x^{T}$, we obtain the following equivalent condition

$$
f(\xi)+g(\xi)^{T} k_{2, \alpha i}>0
$$


The decision parameter $k_{2, \alpha i}$ chosen in accordance with

$$
k_{2, \alpha i}^{T}=l \frac{|f(\xi)| g(\xi)^{T}}{\|g(\xi)\|^{2}}, \quad l>1 \text { and } 0 \neq \xi \in \mathcal{X}_{j i},
$$

guarantees the inequality (43) and, therefore, the condition (iii) in Theorem 3, too.

Scenario (d): Consider the final scenario, when both (s1) and (s2) occur simultaneously. Then, $A_{\mathrm{cl}, \beta j}$ switches to $A_{\mathrm{cl}, \alpha i}$, while the function $V(x)$ is now nonsmooth at $x=\xi \in \mathcal{X}_{j i}$. Similar lines of argument as in Scenario (c) lead to the conclusion that the condition (iii) of Theorem 3 holds again.

Hence, we conclude that the function $V(x)$ in (30) with state feedback design using (32) and (44) satisfies the conditions in Theorem 3 under all conceivable switching situations. Moreover, following the discussion hitherto, $\dot{V}(x)=2 \lambda_{0} V(x)$, almost everywhere, implying

$$
V(x)=V_{0} e^{2 \lambda_{0} t},
$$

where $V_{0}=V(x(0))$. In addition, for any $t>0$ and some $0<M \leq \lambda_{\min }\left(\sum_{i=1}^{n} w_{i} w_{i}^{T}\right)$

$$
M\|x\|^{2} \leq \sum_{i=1}^{n}\left(w_{i}^{T} x\right)^{2} \leq n \max \left(w_{i}^{T} x\right)^{2}=2 n V_{0} e^{2 \lambda_{0} t},
$$

which brings us to the following main result.

Theorem 4. The controlled switched system (29) with arbitrary switching and feedback control design using (32) and (44) is globally exponentially stable.

Finally, it is important to note that for this control scheme, the closed loop stability of the individual subsystems is not mandatory for the stability of the switched system.

Example 3: Consider the system with arbitrary switching between the two-input linear subsystems

$$
A_{1}=\left(\begin{array}{rrr}
-1 & 2 & 3 \\
-3 & 4 & 2 \\
2 & 4 & 5
\end{array}\right), A_{2}=\left(\begin{array}{rrr}
-1 & 2 & 1 \\
-1 & 4 & 2 \\
2 & 4 & 3
\end{array}\right)
$$

with the corresponding input vectors as defined in (29)

$$
b_{1,1}=\left(\begin{array}{c}
1 \\
1.8 \\
2
\end{array}\right), b_{2,1}=\left(\begin{array}{c}
1 \\
0.8 \\
1
\end{array}\right), b_{1,2}=\left(\begin{array}{l}
2 \\
1 \\
1
\end{array}\right), b_{2,2}=\left(\begin{array}{c}
1 \\
-2 \\
1
\end{array}\right) \text {. }
$$

Note that both subsystems $A_{1}$ and $A_{2}$ are unstable, with the eigenvalues located at $-1.72,2.89,6.83$ and $-0.81,0.39,6.42$, respectively. Consider the three independent eigenvectors $w_{1}=[1,0,0]^{T}, w_{2}=[0,1,0]^{T}$, and $w_{3}=[0,0,1]^{T}$, representing the left eigenvectors in our design procedure, with the associated eigenvalue $\lambda_{0}=-2$. Let an initial condition $x(0) \in \mathbb{R}^{3}$ be given, such that $V(x(0))=\frac{1}{2}\left(w_{2}^{T} x(0)\right)^{2}$. Further, let the closed loop be in the $A_{\mathrm{cl}, 12}$ mode, i.e. it is assigned the left eigenvector $w_{2}$. The corresponding vector $k_{2,12}$ is initialized at zero, i.e. $k_{2,12}=[0,0,0]^{T}$. As a consequence, the corresponding vector $k_{1,12}$, computed from (32), reads $k_{1,12}=$ $[1.67,-3.33,-1.11]^{T}$.

According to the elaborations in this section, the Lyapunov function $V(x)=\frac{1}{2}\left(w_{2}^{T} x\right)^{2}$ is a strictly decreasing function in time, until an autonomous or switching control event occurs. Suppose a switching control action occurs upon hitting the boundary manifold at a point $\xi \in\left\{x ;\left(w_{2}^{T}-w_{1}^{T}\right) x=0\right\} \subseteq \mathcal{X}_{21}$. Referring to (43), the following terms can be computed at such a switching point

$$
f(\xi)=\xi^{T}\left(\begin{array}{lll}
1.87 & -0.27 & 2.27 \\
1.87 & -0.27 & 2.27 \\
5.29 & -0.76 & 6.42
\end{array}\right) \xi
$$

and

$$
g^{T}(\xi)=[0.67,0.67,1.89]\left(\xi \xi^{T}\right) .
$$

The corresponding updates for the controllers $k_{2,11}$ and $k_{1,11}$ are computed using (44) and (32), respectively. Note that the Lyapunov function now switches to $V=\frac{1}{2}\left(w_{1}^{T} x\right)^{2}$.

Now consider an autonomous switching event, e.g. from $A_{1}$ to $A_{2}$. The closed loop left eigenvector $w_{1}$ need not be updated due to this switching event. Therefore, $k_{2,21}=$ $k_{2,11}$ remains unchanged. However, in accordance with (32), $k_{1,11}$ switches to $k_{1,21}=[-0.5-1-0.5]^{T}-0.5 k_{2,21}$. The control action is updated by following similar rules upon any further autonomous or switching control event.

\section{CONCLUSION}

Two scenarios for exponential stabilization of linear switching control systems based on a left eigenstructure assignment have been investigated. In the first scenario, we propose a sufficient stabilizability condition for the class of the single-input systems with fixed switching constraints in the state space. Therefore, a single left eigenvector is appropriately constructed to guarantee the simultaneous stabilization of all linear sub-systems, while avoiding the intersection of its corresponding invariant hyperplane with the given switching manifolds in the state space. In the second scenario, the stabilizability of two-input systems under arbitrary switching signals is considered. Therefore, for arbitrary $n$ linearly independent vectors in $\mathbb{R}^{n}$, a nonsmooth common quadratic Lyapunov function is proposed, and a feedback control is designed to guarantee the exponential stability of the Filippov solutions. Thereby, the controller is appropriately parametrized to avoid the sliding modes, while the stability of the constituent sub-systems has not been mandatory.

\section{REFERENCES}

[1] R.Shorten, F. Wirth, O. Mason, K. Wulff, C. King, "Stability criteria for switched and hybrid systems" SIAM Rev., Vol.49, No.4, pp. 545-592, 2007.

[2] A.P. Molchanov, E.S. Pyatniskii, Criteria of asymptotic stability of differential and difference inclusion encountered in control theory, Syst. Con. Let., 13, 1989.

[3] B. D. Schutter, W. P. M. H. Heemels, "Modeling and control of hybrid sytems", Lecture notes of the DISC course, 2004.

[4] E. Feron, Quadratic Stabilizability of Switched Systems via state and output feedback, Technical report CICS-P-468, Center for interlligent control systems, MIT cambridge, 1996.

[5] Y.Kouhi, N. Bajcinca, On the left eigenstructure assignment and state feedback design, American control Conference, 2011.

[6] N.Bajcinca, Y.Kouhi, Yet another pole placement formula, submitted in Automatica, 2011.

[7] T. Kailath, Linear Systems, Prentice Hall, Englewood Cliffs, NJ, 1980.

[8] J.Cortes, "Discountinuous Dynamical Systems". IEEE Control Systems Magazine, 2008.

[9] F. H. Clarke, Y. Ledyaev, R. J. Stern, P. R. Wolenski, Nonsmooth Analysis and Control Theory, New York: Springer-Verlag, 1998. 